\title{
Ukrainian Grasslands Database
}

\author{
Anna Kuzemko
}

\begin{abstract}
The Ukrainian Grasslands Database (GIVD ID EU-UA-001) includes relevés of herbaceous vegetation of the Forest and Forest-Steppe zones of the plain part of Ukraine, carried out by 22 authors between 1932 and 2010. Most are relevés of Anna Kuzemko (998 entries), Lev Balashov (435), Olexander Kuzyarin (391), and Danylo Afanasiev (338). The relevés were established in the 18 administrative regions of Ukraine, the largest number of relevés are from Chernihiv (563 entries), Lviv (420), Kiev (413), Poltava (387) regions. The plot size ranged from 16 to $100 \mathrm{~m}^{2}$. The nomenclature is given in accordance to the "Russia" species list which is based on S. Cherepanov (1995) vascular plants checklist, M. Ignatov \& O. Afonina checklist (1992) for mosses and the lichens identification guides of the USSR (1971-1978) and Russia $(1996,1998)$ for lichens. The database was used to develop the classification of grassland vegetation of the Forest and Forest-Steppe zones of the plain part of Ukraine using the JUICE program. It was identified that the relevés of the database include seven classes of vegetation: Molinio-Arrhenatheretea (2,122 relevés), FestucoBrometea (390), Phragmito-Magno-Caricetea (318), Parvo-Caricetea (197), Koelerio-Corynephoretea (90), Festuco -Puccinellietea (33), Calluno-Ulicetea (31).
\end{abstract}

Keywords: forest zone; forest-steppe zone; heath; meadow; steppe.

\begin{tabular}{|c|c|}
\hline GIVD Database ID: EU-UA-001 & Last update: $2012-05-06$ \\
\hline \multicolumn{2}{|c|}{ Ukrainian Grasslands Database } \\
\hline \multicolumn{2}{|c|}{$\begin{array}{l}\text { Scope: The database includes phytosociological relevés of different types of grasslands from the Forest and Forest-Steppe Zones of Ukraine. } \\
\text { Criteria for inclusion are: plot size from } 16 \text { to } 100 \mathrm{~m}^{2} \text { and clear geographic location. }\end{array}$} \\
\hline Status: completed and continuing & Period: $1932-2010$ \\
\hline \multicolumn{2}{|c|}{ Database manager(s): Anna Kuzemko (anya_meadow@mail.ru) } \\
\hline \multicolumn{2}{|c|}{ Owner: (private) } \\
\hline \multicolumn{2}{|l|}{ Web address: $[\mathrm{NA}]$} \\
\hline Availability: free upon request & Online upload: no Online search: no \\
\hline Database format(s): TURBOVEG & Export format(s): TURBOVEG, Excel \\
\hline \multicolumn{2}{|l|}{ Publication: $[N A]$} \\
\hline Plot type(s): normal plots & Plot-size range: $16-100 \mathrm{~m}^{2}$ \\
\hline Non-overlapping plots: 3,181 & Estimate of existing plots: $5,000 \quad$ Completeness: $64 \%$ \\
\hline Total plot observations: 3,181 & Number of sources: 10 \\
\hline \multicolumn{2}{|l|}{ Countries: UA: $100.0 \%$} \\
\hline \multicolumn{2}{|c|}{ Forest: $0 \%$ — Non-forest: aquatic: 0\%; semi-aquatic: $16 \%$; arctic-alpine: $0 \%$; natural: $0 \%$; semi-natural: $84 \%$; anthropogenic: $0 \%$} \\
\hline \multicolumn{2}{|c|}{ Guilds: all vascular plants: $100 \%$; bryophytes (terricolous or aquatic): $8 \%$; lichens (terricolous or aquatic): $2 \%$} \\
\hline \multicolumn{2}{|c|}{ Environmental data: altitude: $100 \%$; slope inclination: $13 \%$; surface cover other than plants (open soil, litter, bare rock etc.): $62 \%$} \\
\hline \multicolumn{2}{|c|}{ Performance measure(s): cover: $100 \%$} \\
\hline \multicolumn{2}{|c|}{ Geographic localisation: GPS coordinates (precision $25 \mathrm{~m}$ or less): $3 \%$; point coordinates less precise than GPS, up to $1 \mathrm{~km}: 97 \%$} \\
\hline $\begin{array}{l}\text { Sampling periods: } 1930-1939: 2.8 \\
15.2 \% ; 2000-2009: 40.0 \% ; 2010-20\end{array}$ & 349: 1.3\%; 1950-1959: 8.4\%; 1960-1969: 15.1\%; 1970-1979: 11.4\%; 1980-1989: 2.8\%; 1990-1999: \\
\hline Information as of 201 & Irther details and future updates available from http://www.givd.info/ID/EU-UA-001 \\
\hline
\end{tabular}

Anna Kuzemko (anya_meadow@mail.ru)

National Dendrological Park "Sofiyvka" NAS of Ukraine, 12a Kyivska str, 20300 Uman', UKRAINE 